\title{
Estado nutricional y determinantes sociales en niños entre 0 y 5 años de la comunidad de Yunguillo y de Red Unidos, Mocoa - Colombia
}

\author{
Nutritional status and social determinants in children between 0 and 5 years old from the community of \\ Yunguillo and "Red Unidos" in Mocoa, Colombia
}

María Emma Vallejo-Solarte'1, Luz Marina Castro-Castro², María del Pilar Cerezo-Correa³

1 Nutricionista Dietista. Gerente General Empresa Water Food Solutions. Universidad Autónoma de Manizales. Manizales, Colombia, e-mail: mariaemmavallejo@gmial.com

2 Terapeuta Respiratoria, Especialista en Gerencia en Salud Pública, Especialista en Auditoria en Salud. Auditora de Calidad Hospital Fronterizo la Dorada- Apoyo a la Gestión del Régimen Subsidiado. Universidad Autónoma de Manizales. Puerto Asís, Colombia. e-mail: Iuzmitan51@yahoo.es

3 Odontóloga, Magister en Salud Publica, Especialista en Investigación y Docencia Universitaria, Especialista en Auditoria en Salud. Docente Asistente Universidad Autónoma de Manizales. Manizales, Colombia. e-mail: mapice@autonoma.edu.co

Fecha de recepción: Junio 9 - 2015

Fecha de aceptación: Marzo 18 - 2016

Vallejo-Solarte ME, Castro-Castro LM, Cerezo-Correa MP. Estado nutricional y determinantes sociales en niños entre 0 y 5 años de la comunidad de Yunguillo y de Red Unidos, Mocoa, Colombia. Univ. Salud. 2016;18(1):113-125. DOI: http://dx.doi.org/10.22267/rus.161801.24

\section{Resumen}

Objetivo: Establecer el estado nutricional de los niños de 0 a 5 años de la comunidad del Resguardo Yunguillo y de Red Unidos del municipio de Mocoa 2014 y su relación con los determinantes sociales. Materiales y métodos: Estudio descriptivo correlacional con muestreo probabilístico para la población de Red Unidos y población total de Yuguinllo. Se aplicaron a los padres, dos cuestionarios adaptados de la Encuesta Nacional de Salud y Nutrición Colombia - ENSIN 2010 y de la Encuesta Nacional de Demografía y Salud -ENDS 2010 y se diseñó un instrumento para el registro de las medidas antropométricas de los niños, su aplicación contó con el consentimiento informado. Resultados: Se evaluaron 117 niños de Yunguillo y 122 de Red Unidos; encontrando desnutrición aguda 1,7\% y 1,6\%, desnutrición global 23,1\% y 14,5\%, retraso en la talla 43,6\% y $24,2 \%$ y obesidad $12,8 \%$ y 9,7\%, respectivamente. Se encontró relación entre el indicador talla/edad y nivel educativo del padre y la pertenencia a programa de alimentación complementaria y el indicador peso/ edad y el sexo. El acceso a servicios públicos, condiciones habitacionales, de educación y ocupación en ambos grupos son deficientes. Conclusiones: Los niños de ambas comunidades presentan problemas de malnutrición reflejados en los porcentajes elevados de desnutrición crónica, sobrepeso y desnutrición global. Se observaron diferencias estadísticas en el indicador talla para la edad al comparar los niños del grupo de Yungillo y los de Red Unidos.

Palabras clave: Estado nutricional; población indígena; poblaciones vulnerables; determinantes sociales de la salud. (Fuente: DeCS, Bireme). 


\begin{abstract}
Objective: To establish the nutritional status of children from 0 to 5 years old from the community of Yunguillo and from "Red Unidos" of the municipality of Mocoa 2014 and its relationship with social determinants. Materials and methods: A descriptive, correlational study with probabilistic sampling for the "Red Unidos" population and total population of Yuguinllo was made. Two questionnaires adapted from the National Survey of Health and Colombia Nutrition - NSHCN 2010 and the National Demographic and Health survey - NDHS 2010 were applied to the parents and an instrument for child anthropometric measures registration was designed. Its application included their informed consent. Results: 117 children of Yunguillo were evaluated as well as 122 from "Red Unidos". It was found that there is $1.7 \%$ and $1.6 \%$ of acute malnutrition, $23.1 \%$ and $14.5 \%$ of global malnutrition, $43,6 \%$ and $24.2 \%$ of delay in the size, and $12.8 \%$ and $9.7 \%$ of obesity respectively. Relationship between the indicator height/age and educational level of the father was found as well as the supplementary feeding programme membership and the indicator weight / age and gender. The access to public services, housing, education and occupation in both groups are poor. Conclusions: Children of both communities have malnutrition problems reflected in the high percentage of chronic malnutrition, overweight and general malnutrition. Statistical differences were observed in the indicator height-for-age when comparing children of the Yungillo group with those from "Red Unidos".
\end{abstract}

Keywords: Nutritional status; indigenous; vulnerable populations; social health determinants. (Source: DeCS, Bireme).

\section{Introducción}

El estado nutricional es el resultante del balance entre la ingesta y las necesidades energéticas y de nutrientes del organismo, lo que expresa distintos grados de bienestar de las personas y que, en sí mismos, son dependientes de la interacción entre la dieta, los factores relacionados con la salud y el entorno físico, social, cultural y económico.1

La malnutrición describe una condición patológica, consecuencia del desequilibrio en el estado nutricional, y puede deberse a un déficit en la ingesta de nutrientes, a un estado de sobre nutrición o a una alteración en la utilización de estos nutrientes en el organismo. Es así como se establece de acuerdo con el exceso o el déficit de nutrientes la clasificación: desnutrición, sobrepeso, obesidad y deficiencia de nutrientes. La malnutrición es, del mismo modo, un factor de riesgo que incrementa la prevalencia de mortalidad y morbilidad en las poblaciones, disminuyendo la capacidad productiva $\mathrm{y}$, en general, la calidad de vida, lo que refleja en elevados costos sociales.2
La magnitud del problema de malnutrición es relevante en salud pública, tanto a nivel nacional como internacional. Sin embargo, las poblaciones "en riesgo" no se descubren con frecuencia. En nutrición y salud pública, las personas consideradas en riesgo de desarrollar malnutrición deberían ser una preocupación prioritaria. La prevención es más factible y costo-eficiente, si se identifican los grupos en riesgo y se comprenden claramente las causas de la malnutrición.3

La historia de la desnutrición ha representado en el mundo uno de los flagelos más relevantes en el desarrollo de la humanidad, tiene un componente genético, raza y etnia, lo que conlleva a que no se alcancen los objetivos personales en la población, incrementando cinturones de pobreza, desigualdades y exclusión por falta de oportunidades en salud, educación, empleo, saneamiento básico y tenencia de tierra; es por esto que se describe cómo los determinantes sociales se han constituido en circunstancias que contribuyen y perpetúan la desnutrición en el mundo.

El estudio de los determinantes sociales de la salud, reconoce elementos de la estructura 
social que condicionan la posición de las personas en la sociedad, que tienen un efecto directo en la distribución de los problemas o de los factores de la salud, por centrar su interés en las diferencias en las condiciones de salud, entre los estratos sociales, tiene como fundamento el análisis de las inequidades en salud, entendida como aquellas diferencias en el estado de salud de las personas que son evitables y por lo tanto, injustas. 4 Bajo este enfoque, diversos investigadores han hecho un esfuerzo considerable para explicar cómo las desigualdades sociales se convierten en enfermedades y problemas de salud. Este intento es quizás el de mayores retos para la ciencia, ya que se trata de establecer los mecanismos a través de los cuales los individuos "encarnan" las desigualdades de la sociedad que habitan.5

Es así como hoy se conoce que la salud materna y el desarrollo fetal son determinantes críticos de la salud durante la adultez, ejemplo de ello es relación entre el bajo peso al nacer y el riesgo posterior de enfermedad cardiovascular.6

Los determinantes sociales juegan un rol critico en las fases tempanas del desarrollo durante la concepción, la gestación y el periodo posnatal siendo etapas sensibles para el desarrollo cerebral y biológico, que continúan durante la niñez y la adolescencia.7 Es aquí, donde las circunstancias contemporáneas propuestas por Hertzman et al.,8 toman mayor relevancia. Estos autores proponen que estas circunstancias pueden ser organizadas de acuerdo a tres niveles de agregación social: macro, meso y micro.

El macro ambiente socioeconómico nacional, donde el principal determinante de la salud es el ingreso per cápita y su distribución equitativa. El meso o nivel de la sociedad civil que incluye una serie de factores como redes, normas y realidades sociales que posibilitan la cohesión y la capacidad de las instituciones sociales para responder a las necesidades humanas y cambiantes y el micro o determinantes sociales de la salud, asociados con las personas.

Un informe de Naciones Unidas y el Programa Mundial de Alimentos-PMA, reconoce a América Latina y el Caribe por su diversidad, riqueza y contrastes y su producción suficiente para alimentar a sus 550 millones de habitantes. No obstante, cerca de 52 millones de personas no tienen acceso a alimentos suficientes para llevar una vida activa y sana. Además, nueve millones de niños y niñas menores de cinco años sufren de desnutrición crónica. Para estos niños, en su mayoría indígenas y afro-descendientes, la pobreza, el hambre y la pérdida de oportunidades, siguen siendo factores determinantes de su vida cotidiana. Se estima que al menos otros nueve millones de niños están en riesgo de desnutrirse. 9

Si hay algo efectivo en la guerra contra la pobreza y en la erradicación de la desnutrición infantil, es garantizar que los niños y niñas menores de tres años, reciban una adecuada nutrición, puesto que es el periodo más crítico en el desarrollo psíquico y psicomotor.

La Red Unidos es una estrategia de intervención integral y coordinada de los diferentes organismos y niveles del Estado, con el objetivo de generar, construir y fortalecer en el millón y medio de familias que se encuentran en situaciones tanto de pobreza extrema como de desplazamiento, la capacidad de gestionar su propio desarrollo, para mejorar sus condiciones de vida, a través de: (i) Integrar la oferta de servicios sociales para hacerlos coincidir alrededor de la familia de manera simultánea, (ii) brindar transitoriamente, acompañamiento familiar y acceso preferente para asegurar que los recursos e intervenciones permitan superar condiciones mínimas de calidad de vida que no están cubiertas, y (iii) generar corresponsabilidad con las familias para que éstas se comprometan con la superación de su 
situación. Articula los esfuerzos de 18 entidades nacionales, y los focaliza en los dos quintiles más bajos del nivel 1 del SISBEN (82\% de las familias vinculadas), su estructura permite generar los flujos de recursos y de información necesarios para lograr los objetivos propuestos. De esta forma, los tres niveles de gobierno (Nación, Departamentos y Municipios) son corresponsables por la implementación de esta estrategia a nivel territorial.

El pueblo Inga está compuesto por 15.450 indígenas, entre ellos 7.725 son hombres y 7.725 mujeres. La mayoría de ellos, el 62,4\% habita en el departamento de Putumayo, específicamente en el Valle del Sibundoy, a 2.200 metros sobre el nivel del mar. Son reconocidos principalmente por su habilidad para las artesanías, sus conocimientos chamánicos, poderes curativos $\mathrm{y}$ alto nomadismo. En el Putumayo se encuentran ubicados en cuatro comunidades: Osococha, Tandarido, San Carlos y Yunguillo.

Las condiciones sociales y económicas que rodean a estas poblaciones se consideran de alto riesgo para que los niños sufran alteraciones en su salud y en su estado nutricional, especialmente en territorios habitados por comunidades indígenas, por tal razón esta investigación pretendió determinar el estado nutricional de los niños entre 0-5 años pertenecientes al Resguardo indígena de Yunguillo y los niños de 2 a 5 años inscritos en la Red Unidos del Municipio de Mocoa departamento del Putumayo y su relación con los determinantes sociales de la salud, durante el año 2014.

\section{Materiales y métodos}

El estudio corresponde a un descriptivo de alcance correlacional. La población estuvo constituida por 150 niños entre los cero y los cinco años de la comunidad Inga del resguardo Yunguillo según el censo del 2012 registrado en la OZIP (Organización de Indígenas del Putumayo) y 689 niños entre cero y cinco años de Red Unidos, según su base de datos.

\section{Muestra}

Para la población de Red Unidos se realizó un muestreo aleatorio simple y se calculó una muestra con una confiabilidad del 95\%, un margen de error del 3,5\% y se tomaron en cuenta los estimadores de la ENSIN 2010,4 de retraso en la talla, desnutrición global, obesidad y sobrepeso. Se escogió finalmente el estimador de obesidad que garantizara el tamaño de muestra que se ajustara a la investigación, obteniendo una muestra final de 124 niños.

Para los niños indígenas del resguardo Yunguillo se pretendía evaluar toda la población, pero se descartaron aquellos de los cuales no se pudo obtener el consentimiento informado, quedando así 117 niños indígenas, de tal manera que, considerando ambos grupos se evaluaron en total 241 niños. Se excluyeron los niños inscritos en Red Unidos, que fueran indígenas.

\section{Instrumentos y procedimiento}

Las investigadoras diseñaron dos cuestionarios adaptados de la Encuesta Nacional de Salud y Nutrición Colombia ENSIN 2010 y la Encuesta Nacional de Demografía y Salud -ENDS 2010. Se realizó una prueba piloto con diez padres de familia de las comunidades indígenas y como resultado de ésta, se realizaron los ajustes necesarios. Se diseñó el instrumento para el registro de las medidas antropométricas.

\section{Procedimiento}

a) Presentación de la investigación y concertación para su realización con los líderes del resguardo indígena y con las directivas de la Red Unidos del Putumayo. b) Capacitación a un docente y a un promotor de salud indígena para aplicación de encuestas a padres y/o cuidadores en comunidad indígena y a dos auxiliares de enfermería para la 
población de Red Unidos. c) Explicación de los objetivos del proyecto a los padres y cuidadores de los niños y solicitud del consentimiento informado. d) Toma las medidas antropométricas a los niños seleccionados por parte de una nutricionista con experiencia.

La técnica utilizada para la toma de las medidas antropométricas fue la descrita en la Resolución 2121 del Ministerio de Salud de Colombia. Se calibraron los equipos en forma periódica. e) Aplicación de los cuestionarios a los padres o responsables de los niños valorados.

\section{Procesamiento y análisis}

La información se digitó en el programa estadístico SPSS Versión 21 previo diseño de la base de datos. Se utilizó además el STATHGRAFHICS, con licencia de la Universidad Autónoma de Manizales.

Los momentos durante el análisis fueron: a) Análisis descriptivo de los determinantes en cada uno de los grupos. b) Comparación del estado nutricional del grupo de indígenas y el grupo de Red Unidos c) Análisis bivariado entre estado nutricional y los determinantes sexo, ingreso, seguridad alimentaria, nivel educativo de la madre y del padre) y d) Análisis de conglomerados para identificar grupos con características similares.

\section{Consideraciones éticas}

Esta investigación se acogió a lo descrito en la Resolución 008430 de 1993 del Ministerio de Salud de Colombia,10 y obtuvo aprobación por el Comité de Ética de la Universidad Autónoma de Manizales. Se solicitó consentimiento informado a los padres o cuidadores de los niños para responder la encuesta y para la toma de los indicadores antropométricos a los niños. Además, se solicitó consentimiento al líder del resguardo de Yunguillo y a las directivas de Red Unidos en el municipio.

\section{Resultados}

Los indígenas del resguardo Yunguillo están ubicados en cuatro comunidades: Osococha, Tandarido, San Carlos y Yunguillo. El 51,3\% son mujeres, el $40 \%$ son menores de 2 años y el $100 \%$ está afiliado al Sistema General de Seguridad Social en Salud, mediante el régimen subsidiado.

El $77 \%$ pertenece a familias nucleares, con un promedio de 7 personas por hogar. Los padres en un 50\% están en unión libre. El 56\% de los padres y el $44 \%$ de las madres tienen un nivel educativo de primaria. La mayoría de las madres son amas de casa y los padres se dedican a la agricultura. Más del $90 \%$ en las cuatro comunidades perciben ingresos inferiores a un salario mínimo. Los padres de los niños de las comunidades de Osococha y Yunguillo reciben promedios más altos de ingresos, el padre que más ingresos recibe es \$ 1.080.000 y refieren que los ingresos en una mayor proporción se destinan para la alimentación.

Se identificó que el acceso a servicios públicos es muy limitado. El abastecimiento de agua a través del acueducto público lo tiene una proporción inferior al $10 \%$ en las cuatro comunidades. En San Carlos ninguno cuenta con servicio sanitario y en Tandarido el 90,5\%. En Yunguillo el 9,3\% refiere que las basuras las recoge el servicio de aseo, la mayoría en todas las comunidades tira la basura a un lote o zanja.

En todas las comunidades las casas tienen en promedio tres cuartos, la más grande, nueve y la más pequeña un solo cuarto. La mayoría de las casas tiene el techo en zinc, los pisos y las paredes en madera. Todos los padres afirman que tienen un cuarto que utilizan para cocinar. En los hogares se cultiva algún tipo de productos, en un $86,4 \%$ en Osocoha, el $100 \%$ en San Carlos, el $95,5 \%$ en Tandarido y el $97,7 \%$ en Yunguillo; los alimentos que producen son los típicos de la región como el 
banano, yuca, maíz, plátano, chiro y productos de la chagra. La mayor proporción los utiliza para el consumo. En la vereda de Tandarido $54,5 \%$ de las personas refiere haber recibido educación en temas de nutrición, en las demás la proporción es inferior al 40\%.

Con respecto los resultados del cuestionario de "Frecuencia de Consumo" utilizado por la ENSIN-2010, se identificó que el $86 \%$ de personas reconoce haber consumido el día anterior el chocolate y el agua de panela, el $81 \%$ los tubérculos y los plátanos, el $72 \%$ el huevo y el $31 \%$ carnes rojas, porcentajes inferiores a estos reconocen haber consumido verduras, leche en polvo y derivados de la leche.

Los resultados de la aplicación de la escala de seguridad alimentaria indicaron que el 66\% está en seguridad alimentaria, el $33 \%$ en inseguridad alimentaria leve y el $1 \%$ en inseguridad alimentaria moderada.

Según los resultados de las medidas antropométricas, en el resguardo de Yunguillo se encontró que el $3,4 \%$ de la población presenta desnutrición aguda, el 43,6\% destrucción crónica, el 23,1\% desnutrición global; el $12,8 \%$ presentó obesidad y $27,4 \%$, sobrepeso.

En relación a los niños pertenecientes a Red Unidos, EL 57,3\% está en el grupo de 2 a 5 años de edad; el promedio de edad es de 29,3 meses, el 94,4\% está afiliado al régimen subsidiado de salud, 3,2\% al contributivo y el restante, al especial. Según las características familiares, se identificó que el $40 \%$ de los niños conviven en familias nucleares completas y el 33\% en familias nucleares incompletas.

La mayor proporción de los padres se encuentra en unión libre $50 \%$, seguido por casado $34 \%$. El 55,6\% de los padres y el $43,6 \%$ de las madres cursaron hasta primaria. El 82\% de las madres se dedica a las actividades del hogar.
El ingreso promedio mensual de las familias es de $\$ 476.333$. El $78,2 \%$ recibe menos de un salario mínimo legal vigente. Los padres refieren en su mayoría, que los ingresos en una mayor proporción se destinan para la alimentación.

Los niños de Red Unidos en un 98,4\% tienen acceso a energía, siete de cada diez tiene acceso a acueducto y recolección de basuras. Los servicios públicos que llegan a una menor proporción de la población son el alcantarillado y el gas por tubería. En cuanto al servicio sanitario el $84,7 \%$ informa que este es de uso exclusivo; la mayor proporción usa el inodoro conectado a alcantarillado y conectado a pozo séptico.

El material que predomina en el techo de las viviendas es el eternit 46,8\% y el zinc 39,5\%; el piso en cemento $60,5 \%$; en las paredes refiere tener ladrillo $41,1 \%$ y bloque $28,2 \%$. El $80,6 \%$ de los padres afirma que tiene un cuarto que utiliza para cocinar, los demás refieren que utilizan un cuarto para cocinar y dormir. Para la mayoría este cuarto es de uso exclusivo de las personas del hogar 89,5\%. Utiliza como combustible para cocinar el gas en cilindro $82,3 \%$, seguido por la leña $6 \%$.

El 24,2\% cultiva alimentos en el hogar, entre los de mayor producción están los típicos de la región como el banano, yuca, plátano, chiro y yota; la mayoría, para consumo en el hogar.

En el Cuestionario de Frecuencia de Consumo se identificó que el $81 \%$ de las personas reconoce haber consumido el día anterior el agua de panela, el 66\% huevo, el 65\% jugo de frutas, el $64 \%$ tubérculos y plátanos, el $52 \%$ carnes rojas, el $51 \%$ dulces y en porcentajes inferiores, los otros alimentos.

Según la escala de seguridad alimentaria, el $61 \%$ está en seguridad alimentaria, el 38\% inseguridad alimentaria leve, el $0,8 \%$ en inseguridad alimentaria moderada y el 0,8\% inseguridad alimentaria severa.4 En la 
evaluación nutricional de los niños de Red Unidos se encontró una desnutrición aguda de encontró que el 9,7\% presentó obesidad y el $23,4 \%$ sobrepeso.

$3,2 \%$, retraso en talla $24,2 \%$, y desnutrición

global $14,5 \%$. En cuanto a exceso de peso, se

Tabla 1. Distribución porcentual de los indicadores antropométricos. Niños indígenas de Yunguillo y ONG Red Unidos, Mocoa - Putumayo

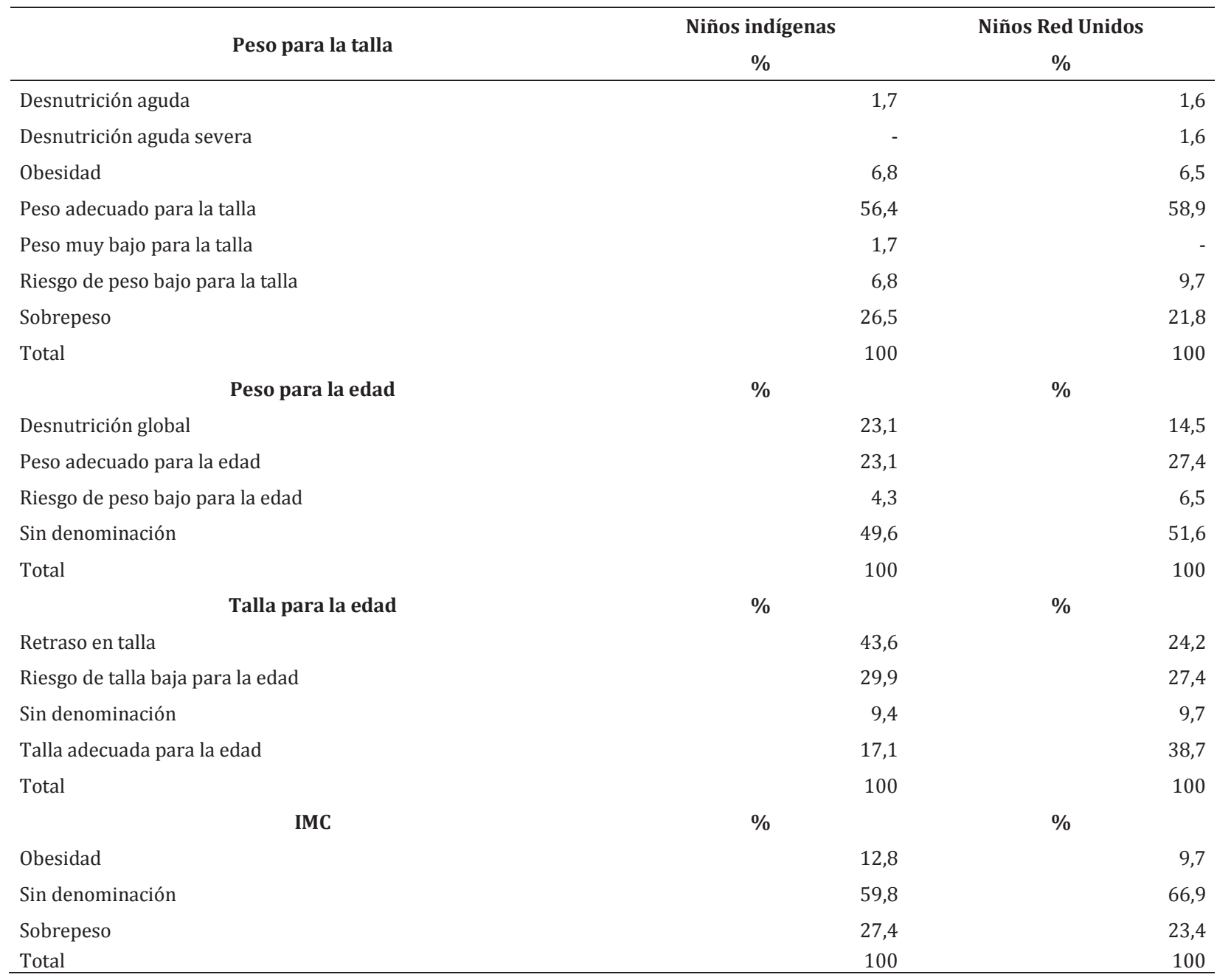

Se encontró relación entre el indicador talla para la edad y el pertenecer al grupo de indígenas o al grupo de Red Unidos (chi= 16,843, p: 0,001). Los demás indicadores no mostraron relación con el grupo al cual pertenecen los niños. Se encontró relación estadística entre el indicador talla para la edad y el nivel educativo del padre $(\mathrm{chi}=43,3 \mathrm{p}=$
$0,00)$ y que pertenecía a programa de alimentación complementaria (chi=12,5 p= $0,00)$ y entre el indicador peso para la edad y el sexo (chi=9,19 $p=0,02)$. Con el fin de identificar grupos con características similares, se realizó un análisis de conglomerados y se identificaron dos conglomerados o clases. 
Grafico 1. Clases de niños con características similares según la clasificación jerárquica

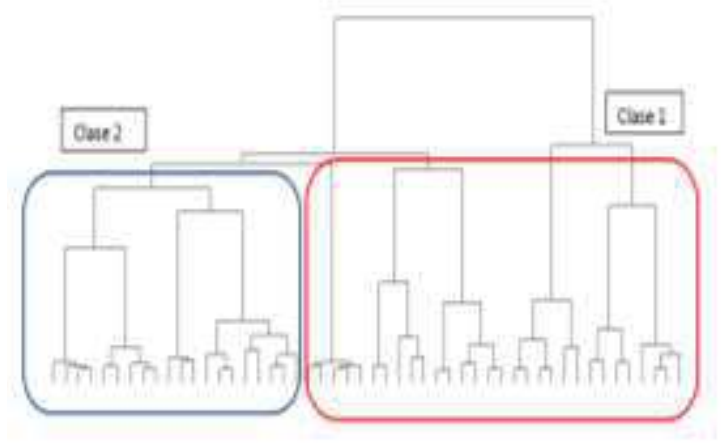

La clase uno más grande y homogénea, está constituida por el $60 \%$ de los niños. Se caracteriza porque los niños pertenecen a Red Unidos, el nivel educativo del padre y de la madre es básica secundaria, tienen talla adecuada para la edad y peso adecuado para la edad.

La clase dos está constituida por el $40 \%$ de los niños. Se caracteriza porque son de Yunguillo, el nivel educativo de la madre es primaria y del padre primaria incompleta y tiene retraso en la talla.

\section{Discusión}

En esta investigación se encontró que los niños de la comunidad indígena presentan mayores proporciones de malnutrición que los niños pertenecientes a Red Unidos.

Otras investigaciones también han evidenciado la malnutrición en los niños indígenas. En niños Arhuacos menores de 5 años de edad encontraron que el 91,1\% están con retraso para la talla, $10 \%$ con desnutrición aguda, 5,3\% con desnutrición aguda severa, $8,8 \%$ con obesidad y el $10 \%$ se catalogaron con factor de riesgo para el neurodesarrollo. Al procesar y graficar estos datos se evidencia la tendencia de la población en general a la talla baja con desviación hacia el sobrepeso en los indicadores de peso para la talla e índice de masa corporal para la edad.11

Otro estudio mostró que el comportamiento acumulativo de la desnutrición crónica en función de la edad durante el período de crecimiento de los niños Embera, puede indicar que el ambiente alimentario adverso acompaña la vida indígena. En este estudio se observó un aumento de la desnutrición crónica con la edad, de 48,4\% en los niños y niñas de 0-2 años a 76,6\% entre 2 y 5 años y, finalmente a $77,1 \%$ de talla baja en los jóvenes (10-19 años). A medida que el niño indígena se separa de los cuidados maternos, experimenta la sustitución de la leche materna por alimentos de baja calidad nutricional y se profundiza el retraso del crecimiento y el aumento de desnutrición crónica.12 Este último hallazgo consistente con los resultados de la ENSIN 2010,4 mostró que la proporción de niños indígenas con sobrepeso, fue significativamente mayor $(6,8 \%)$ que la de los afrodescendientes $(4,0 \%)$.

En esta investigación se encontró relación estadística entre el indicador talla para la edad y el nivel educativo del padre y la pertenencia a programa de alimentación complementaria y entre el indicador peso para la edad y el sexo. La literatura evidencia que existen determinantes sociales que podrían influenciar el desarrollo del sobrepeso y la obesidad, como son la educación, el sexo, la pobreza y el lugar de residencia. En la ENSIN 2010,4 los niños con prevalencias mayores de obesidad fueron hijos de madres con mayor nivel educativo y con niveles de SISBEN 4. Acosta,13 comparó los resultados de la ENSIN 2005 y 2010 encontrando una relación inversa entre la obesidad y el nivel socioeconómico y el nivel educativo de las mujeres.

Otros autores como Álvarez,14 también han estudiado la relación entre el nivel socioeconómico, el nivel educativo y la obesidad encontrando diferencias de acuerdo con las condiciones socioeconómicas al 
interior de municipios, como es el caso de Medellín, donde se encontró mayor porcentaje de población con obesidad en los estratos medio y bajo, así como mayor prevalencia en las personas con menor nivel educativo y en quienes provienen de familias cuyos ingresos son menores a dos salarios mínimos.

Al respecto Fortich,15 refiere que la educación está asociada inversamente al sobrepeso y la obesidad y por el contrario, la riqueza está directamente asociada con el sobrepeso y la obesidad; así mismo, el sobrepeso es mayor en los hombres que en las mujeres a edades tempranas, y la obesidad es más frecuente en mujeres y es mayor en la zona urbana, debido al incremento del consumo de las "comida rápida" que supone hasta un tercio de calorías consumidas, porque dichos alimentos contienen más calorías, grasas saturadas, colesterol y menos fibra que las comidas caseras.16-18

En esta investigación se identificó que una alta proporción de personas que subsisten con ingresos inferiores al salario mínimo. En diversos estudios, se afirma que las poblaciones indígenas sufren pobreza, no sólo por la falta de propiedad y disponibilidad de la tierra, sino también por la fragmentación de sus economías comunitarias, sus dificultades de inserción laboral, así como por los prejuicios étnico-raciales de los que son víctimas.19,20 Además, los indígenas $\mathrm{y}$ afrodescendientes suelen habitar en tierras de poca calidad productiva, en lugares de difícil acceso y con fuertes carencias de acceso a infraestructuras y servicios básicos de salud, educación y seguridad social, que les afectan negativamente varios determinantes de su salud y específicamente su nutrición.

De acuerdo con la encuesta de calidad de vida sobre el porcentaje de hogares por opinión del jefe o cónyuge sobre los ingresos de su hogar ENCV 2010 - 2011 a nivel de regiones, la percepción más optimista sobre la capacidad que tienen los ingresos para cubrir los gastos mínimos del hogar se encuentra en Bogotá con 4,2 puntos porcentuales por encima del año 2010. San Andrés y Valle, también muestran un mayor porcentaje donde los jefes de hogar o su cónyuge consideraron que los ingresos de su hogar "cubren más que los gastos mínimos", $17,8 \%$ y 16,1\%, respectivamente. La región Pacífica mantiene en el 2011 la calificación más pesimista sobre los ingresos del hogar; el $47,2 \%$ de los jefes de hogar o su cónyuge consideraron que los ingresos de su hogar "no alcanzan para cubrir los gastos mínimos", aunque en este año se registró una disminución de 2,2 puntos porcentuales con respecto a lo expresado en 2010 (49,4\%).21

En la investigación de escolaridad materna y desnutrición del hijo, hace referencia al nivel educativo de los padres como un factor clave para crear un ambiente saludable y potenciar el adecuado crecimiento, desarrollo y así evitar la desnutrición en la primaria infancia. 22

En esta investigación se encontró que la alimentación está basada en su mayoría en consumo de carbohidratos en las dos poblaciones, esto podría explicarse porque los tubérculos son fuente de producción principal en el departamento del Putumayo y de los alimentos de más fácil acceso para la población.

Los resultados con respecto a la obesidad, de este estudio concuerdan con los estudios realizados en niños peruanos, en donde encontraron que a medida que incrementa el nivel de pobreza también aumenta el consumo de carbohidratos y, por el contrario, disminuye el consumo de proteínas, hierro y vitaminas.23

La OMS considera que la obesidad es una epidemia que inicia a edades tempranas con un origen multicausal,24,25 y estima que en el 2015 se incrementará a 2,3 mil millones de personas con sobrepeso y 700 millones de personas obesas.30 En el Perú, el estado nutricional de la población ha tenido un cambio gradual, pues coexisten diferentes formas de malnutrición 
como la desnutrición crónica, el sobrepeso y la obesidad en los niños,26,27 estos últimos han aumentado en forma progresiva debido a los cambios en la dieta y estilos de vida producto de la urbanización y desarrollo económico.28,29

En cuanto a la actividad laboral, la población de Red Unidos en su mayoría predomina el trabajo informal y en la comunidad indígena la mayoría son agricultores, En Colombia,31 la informalidad laboral y empresarial es una de las características de la economía y ha sido identificada como una de las principales barreras para alcanzar mayores niveles de crecimiento económico, debido a sus efectos negativos sobre las condiciones de trabajo, la productividad, y la financiación del Sistema de Protección Social.

La investigación de Larrea et al.,32 demostró que en Ecuador, Perú y Bolivia, tanto los hogares indígenas como en general, las regiones andinas sufren más intensamente la desnutrición. Este efecto negativo se mantiene incluso bajo condiciones similares de educación de la madre, uso de servicios de salud, condiciones de vivienda e infraestructura habitacional (agua, alcantarillado, electricidad).

Los hogares de las regiones andinas, y en particular los hogares indígenas, están más afectados por la desnutrición que los hogares no indígenas y de otras regiones, aún si se compara aquellos que tienen similares condiciones de educación, servicios de salud, vivienda e infraestructura habitacional, y otras variables de control, como la edad y el sexo de los niños.

En consecuencia, la desnutrición en los países no se explica únicamente por determinantes sociales, usualmente asociados a la pobreza, o por el nivel educativo de la madre. Existen elementos relacionados con distintas formas de discriminación a la población indígena y otros elementos regionales que afectan a las zonas andinas. ${ }_{33}$ En los niños de Red Unidos se identificó retraso en la talla en un $24,2 \%$, y en el resguardo de Yunguillo en un 43,6\%, estas se consideran cifras elevadas en comparación con la nacional y la departamental.

Según los patrones de crecimiento de la OMS, el $13,2 \%$ de los niños colombianos presentó retraso en la talla $(-<2 \mathrm{DE})$ y de ellos el $3 \%$ se encontró en el rango de<-3DE. En la Resolución 2121 de adopción de nuevos patrones, se incluyó el rango de -1a- 2 como riesgo de talla baja para la edad, en el cual se esperaría encontrar aproximadamente al 13,4\%de la población (en una distribución normal), sin embargo en el país el 30,2\% de la población menor de cinco años se encuentra en este rango. Considerando el lugar de residencia, el retraso en talla fue de mayor prevalencia en el área rural que en el área urbana (17\% frente 11,6\%).34

El Sistema de Vigilancia Alimentaria y Nutricional SISVAN 2013,35 reporta para el año 2014 en el Putumayo un 14,2\% de niños con desnutrición crónica (talla baja para la edad), siendo los más afectados la población indígena. En esta investigación las cifras fueron aún más elevadas 43,6\% para los niños de Yunguillo y $24,2 \%$ para los niños de Red Unidos.

La investigación titulada Desnutrición Infantil en Colombia: inequidades y determinantes, 36 hizo un análisis para identificar los determinantes de la desnutrición crónica infantil y encontró que los niveles de desnutrición crónica son mayores en la zona rural que en la urbana, y mayores en los hombres que en las mujeres. La desnutrición crónica mostró una relación positiva, bastante marcada, con el nivel socioeconómico, especialmente en las zonas urbanas. Los resultados de esta investigación muestran también que en poblaciones vulnerables como lo son niños del resguardo de Yunguillo y de Red Unidos los problemas nutricionales son evidentes. 
En esta investigación se identificó que la mayoría de los padres se consideran en seguridad alimentaria a pesar de los ingresos bajos y de los resultados de la encuesta de consumo. Según el informe del estado de inseguridad alimentaria en el mundo 2009, se destaca como consecuencia de la crisis alimentaria y económica que los hogares pobres generan mecanismos de adaptación en época crisis y se ven obligados a consumir comidas y alimentos menos nutritivos, reducir los gastos sanitarios y de educación y vender sus bienes.37

En Colombia según la ENSIN 2005 se observan debilidades importantes en las practicas alimentarias, orientadas a obtener una alimentación saludable, las cuales son más acentuadas en el área rural y en los niveles $1 \mathrm{y}$ 2 del SISBEN, prueba de ello es que el 15\% de las personas no refieren consumir huevos o carnes diarias, lo que en términos absolutos correspondería a $6,8 \%$ millones de colombianos, así mismo se presenta una brecha importante, según el nivel del SISBEN, en el que es $10 \%$ menor el consumo de estos alimentos es el SISBEN 1 en comparación con el nivel 4 o más, lo que indica una relación ente las condiciones socioeconómicas y la situación alimentaria y nutricional de la población.

En esta investigación el análisis multivariado mostró que la clase 2 representada por niños de Yunguillo se caracteriza por problemas nutricionales como retraso en la talla, este hallazgo ya ha sido evidenciado en otras investigaciones como la de Sánchez, 38 que señala entre los principales problemas de salud en las comunidades indígenas la desnutrición, los altos índices de morbilidad, el desconocimiento de su cultura médica y la constante dificultad para cubrir a la totalidad de la población con los servicios de salud necesarios. Al igual, el estudio de la gran expedición humana, que describe, la situación nutricional y alimentaria de 26 grupos indígenas y cinco grupos afrocolombianos, muestra que el problema más relevante es la desnutrición crónica de la niñez indígena que está ocasionada por la interrelación de los factores relacionados con la disponibilidad, el consumo y la utilización de alimentos; lo que repercute negativamente en el crecimiento y desarrollo de los niños. 39

\section{Conclusiones}

1. Los niños de 0 a 5 años de la comunidad Inga y de la población de Red Unidos del municipio de Mocoa presentaron problemas de malnutrición reflejados en los porcentajes elevados de desnutrición crónica, sobrepeso y desnutrición global.

2. Los niños de 0 a 5 años de la comunidad Inga presentaron mayor prevalencia de malnutrición en comparación con los niños(as) la población de Red Unidos del municipio de Mocoa.

3. Se observaron diferencias estadísticas al comparar los niños del grupo de indígenas y de Red Unidos, con el indicador talla para la edad.

4. Considerando los dos grupos, los niños indígenas y los niños de Red Unidos se encontró relación estadística entre el indicador talla para la edad y el nivel educativo del padre y la pertenecía a programa de alimentación complementaria y entre el indicador peso para la edad y el sexo.

Según los resultados del estudio se recomienda promover políticas públicas intersectoriales. Desde el enfoque de cosmovisión y determinantes sociales dirigidas a la reducción de la malnutrición desde la prevención, la intervención y el seguimiento.

Conflicto de intereses: Ninguno declarado por los autores. 


\section{Referencias}

1. Nación. MdSdl. Plan Federal de Salud. Encuesta Nacional de Nutrición y Salud, 2004-2005 Argentina.

2. INCAP-OPS-Menchu MT S. Propuesta de indicadores para la Vigilancia Alimentaria y Nutricional. Guatemala.: Publicación INCAP PCE073.; 2012.

3. Lorenzo G. Desnutrición ¿Por qué existe? An Venez Nutr. 2005; 18(1):69-71.

4. ICBF. Encuesta Nacional de la Situación Alimentaria y Nutricional 2010.

5. Álvarez L. Los determinantes sociales de las inequidades en salud y nutrición. In: 12o Simposio Nacional de Nutrición Humana.; 2008; Medellín: Escuela de Nutrición y Dietética, Universidad de Antioquia.; 2008: 77-82.

6. Barker D, Erikson J, Forsén T, Osmond C. Fetal origins of adult disease: strength of effects and biological basis. Int J Epidemiol. 2002; 31:12351239.

7. Richter L. The importance of caregiver-child interactions for the survival and healthy development of young children: a review. Geneva. World Health Organization. 2004.

8. Hertzman C, Power C, Matthews S, Manor O. Using an interactive framework of society and life course to explain self-rated health in early adulthood. Soc. Sci Med. 2001; 53:1575-1585.

9. Alimentos. RyoNU-Pmd. Hacia la erradicación de la desnutrición infantil en América Latina y el Caribe. Oficina Regional del PMA para América Latina y el Caribe.

10. República de Colombia, Ministerio de Salud, Resolución 008430 de 1993. Por la cual se establecen las normas científicas, técnicas y administrativas para la investigación en salud, Bogotá, 1993

11. Arias M, Tarazona MC, Lamus F, Granados C. Estado nutricional y determinantes sociales asociados en niños menores de 5 años de edad Rev. Salud Pública. 15 (4): 565-576, 2013.

12. Rosique J, Restrepo M, Manjarrés L, Gálvez A, Santa J. Estado nutricional y hábitos alimentarios en indígenas Embera de Colombia. Rev Chil Nutr. 2010 Sep; 37(3) 270-280.

13. Acosta K. La obesidad y su concentración según nivel socioeconómico en Colombia. Cartagena: Banco de la República; 2012. [citado mayo de 2013]. Documentos de Trabajo sobre Economía Regional; $170 . \quad$ Disponible en: http://www.banrep.gov.co/es/node/27047.

14. Álvarez-Castaño LS, Goez-Rueda JD, CarreñoAguirre C. Factores sociales y económicos asociados a la obesidad: los efectos de la inequidad y de la pobreza. GerencPolit Salud. 2012; 11:98-110.
15. Fortich R, Gutiérrez J. Los determinantes de la obesidad en Colombia. Economía \& Región. 2011; 5(2):155-82.

16. Ortiz-Moncada R, Alvarez-Dardet C, Miralles-Bueno J, Ruiz-Cantero M, Dal Re-Saavedra M, Villalba C, et al. Determinantes sociales de sobrepeso y obesidad en España 2006. MedClin (Barc). 2011; 137:678-84.

17. Philip J, Leach R, Kalamara E, Shayeghi M. The worldwide obesity epidemic. Obes Res. 2001; 9(4):228S-33S.

18. Magnusson RS. What's law got to do with it Part 2: Legal strategies for healthier nutrition and obesity prevention. Aust New Zealand Health Policy, 2008; 5:11.

19. Psacharopoulos G, Patrinos HA (eds.). Indigenous people and poverty in Latin America: An empirical analysis. Washington, D.C.: The World Bank, 1994.

20. Hopenhayn M, Bello A, Miranda F. Los pueblos indígenas y afrodescendientes ante el nuevo milenio. CEPAL. Naciones Unidas. Santiago de Chile. Abril de 2006.

21. DANE. Encuesta de calidad de vida 2010 - 2011. Colombia.

22. Tejada LM, González de Tineo. Escolaridad materna y desnutrición del hijo o hija. Centro clínico nutricional Menca de Lioni, Caraca. AnVenez 2005, 18 (2): 162, 16869.

23. Chescheir N. Obesidad en el mundo y su efecto en la salud de la mujer. Obstet Gynecol 2011;117:121322.

24. Rojas C, Calderón M, Taipe M, Bernui, Ysla M, Riega V. Consumo de energía y nutrientes, características socioeconómicas, pobreza y área de residencia de niños peruanos de 12 a 35 meses de edad. Rev Peru Med Exp Salud Publica.2004; 21(2):98-106.

25. Low S, Chew Chin M, Deurenberg-Yap M. Review on Epidemic of Obesity. Ann Acad Med Singapore. 2009; 38:57-65.

26. Pérez BM, Landaeta-Jiménez M, Amador J, Vásquez M, Marrodán MD. Sensibilidad y especificidad de indicadores antropométricos de adiposidad y distribución de grasa en niños y adolescentes venezolanos. INCI. 2009; 34(2):84-90.

27. Miranda M, Chávez H, Aramburú A, Tarqui-Mamani C. Indicadores del Programa Articulado Nutricional. Monitoreo Nacional de Indicadores Nutricionales (MONIN 2008-2010-CENAN-DEVAN). Lima: Instituto Nacional de Salud; 2010.

28. Instituto Nacional de Estadística e Informática (INEI). Encuesta Demográfica y de Salud Familiar 2010. Lima: INEI; 2011.

29. Tarqui-Mamani C, Sánchez J. Situación nutricional del Perú, 2010. Informe preliminar. Lima: Instituto Nacional de Salud; 2010.

30. Barria M, Amigo H. Transición nutricional: una revisión del perfil latinoamericano. Arch Latinoam Nutr. 2006; 56(1):3-11. 
31. Colombia. Departamento Nacional de Planeación. (2010). Bases del Plan Nacional de Desarrollo 2010-2014, "Prosperidad para Todos".

32. Larrea C. Desnutrición, Etnicidad y Pobreza en el Ecuador y el Área Andina. Artículo publicado en el libro Barrera, Augusto, et.al. "Foro sobre la democracia, el bienestar y el crecimiento económico". UNICEF-FLACSO- Terranueva, Ecuador, 2006

33. Low $\mathrm{S}$, Chew M, Deurenberg Y. Review on Epidemic of Obesity. Ann Acad Med Singapore.2009; 38 (1):57-65.

34. xxxxEncuesta Nacional de Demografía y Salud ENDS 2010.

35. Sistema de Vigilancia Alimentaria y Nutricional. Sisvan del departamento del Putumayo. 2013

36. Flórez CE, Nupia A. Desnutrición infantil en Colombia: inequidades y determinantes. Documentos CEDE [publicación en línea] 2001 [citado mayo 2014] Disponible en: http://www.bvsde.paho.org/texcom/nutricion/D 2001.pdf

37. Organización Mundial de la Salud. Informe sobre la salud en el mundo 2005. !cada madre y cada niño contaran! Ginebra. 2005. [citado mayo 2014] Disponible en: http://www.who.int/whr/2005/overview_es.pdf

38. Sánchez E, Enrique M, Benjumea S, Rodríguez I, Nieto B. Los pueblos indígenas en Colombia. Derechos, políticas y desafíos. Unicef. Oficina de área para Colombia y Venezuela. Bogotá. 2003.

39. Tobar L, Chinchilla M, Cortés Y, Mondragón A. Estado alimentario y nutricional de comunidades indígenas y poblaciones afrocolombianas. Gran Expedición Humana. Terrenos de la Gran Expedición Humana. Bogotá. Pontificia Universidad Javeriana.; 1996. 